
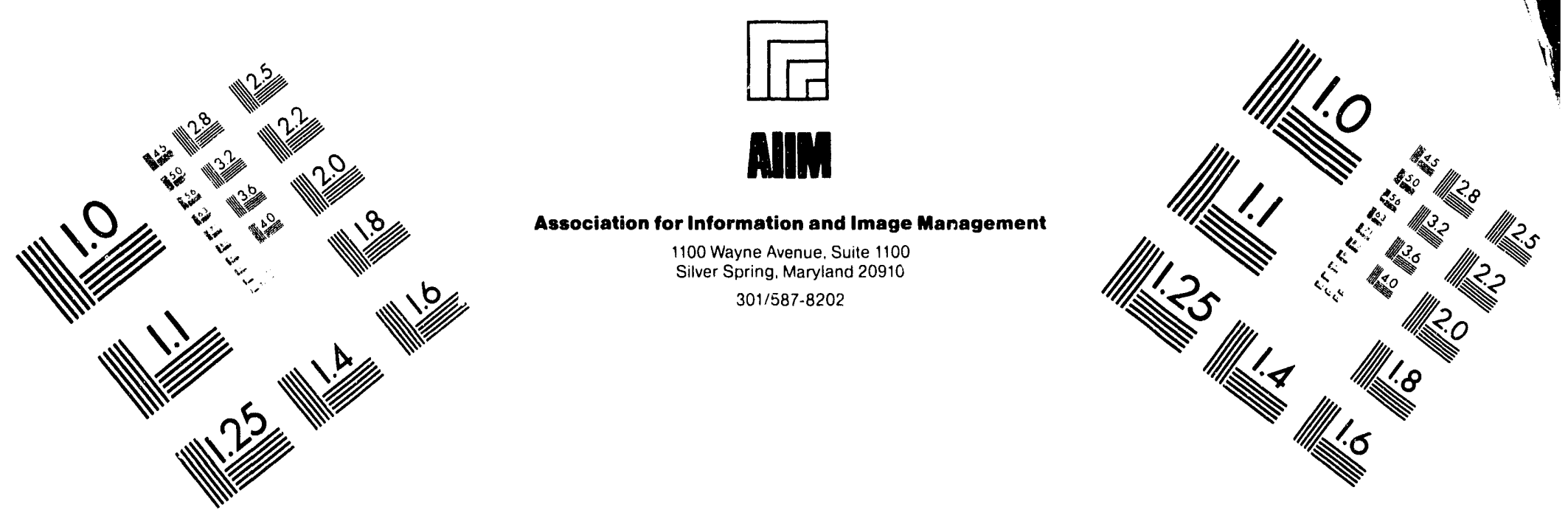

\title{
Centimeter
}

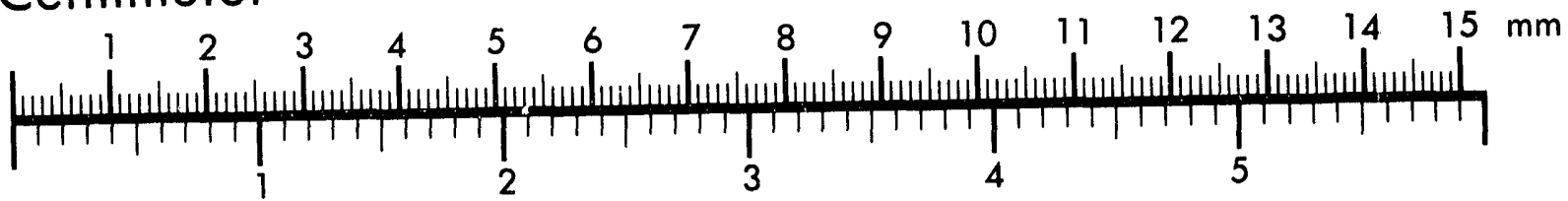
Inches
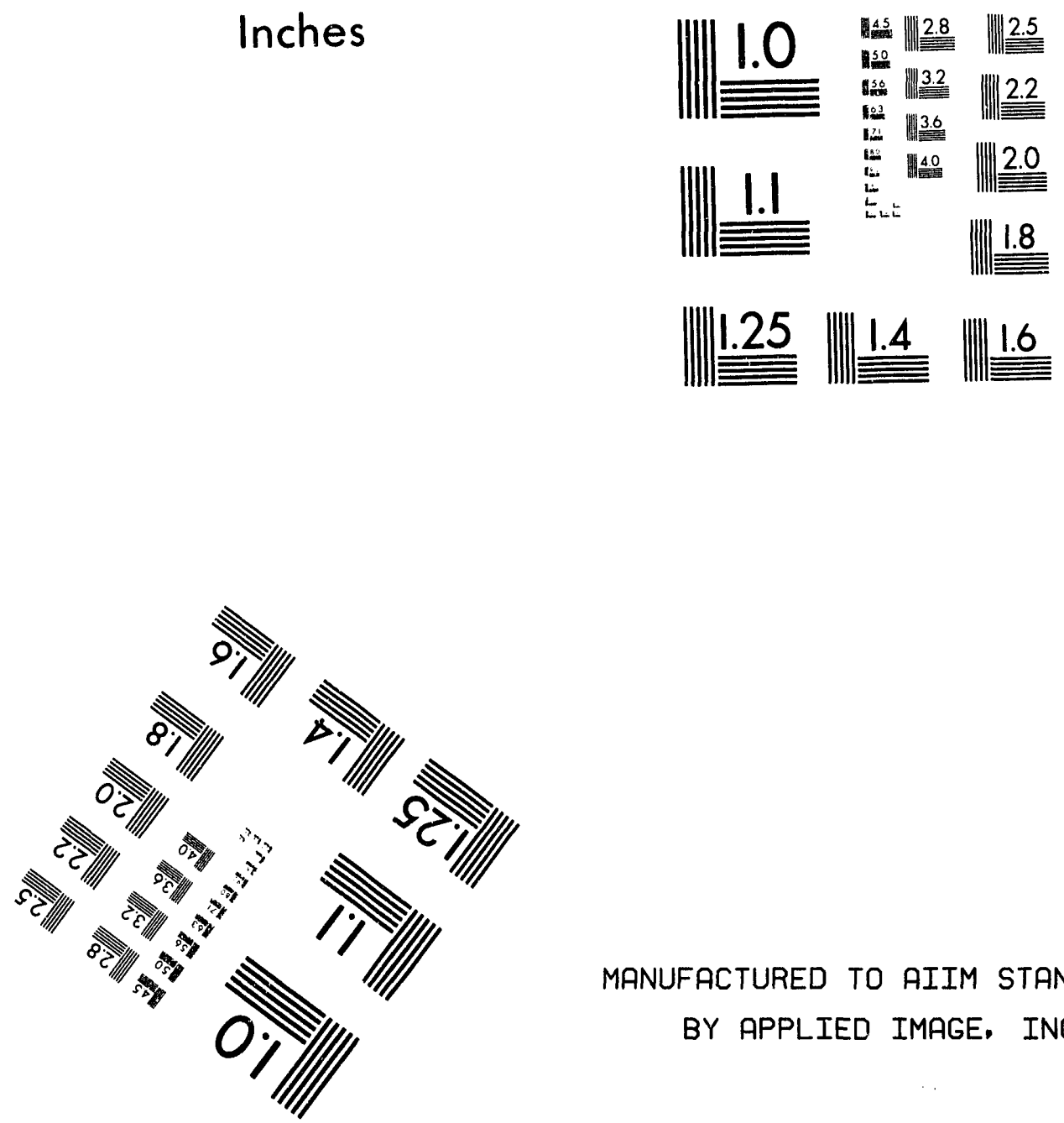

MANUFACTURED TO AIIM STANDARDS BY APPLIED IMAGE, INC.

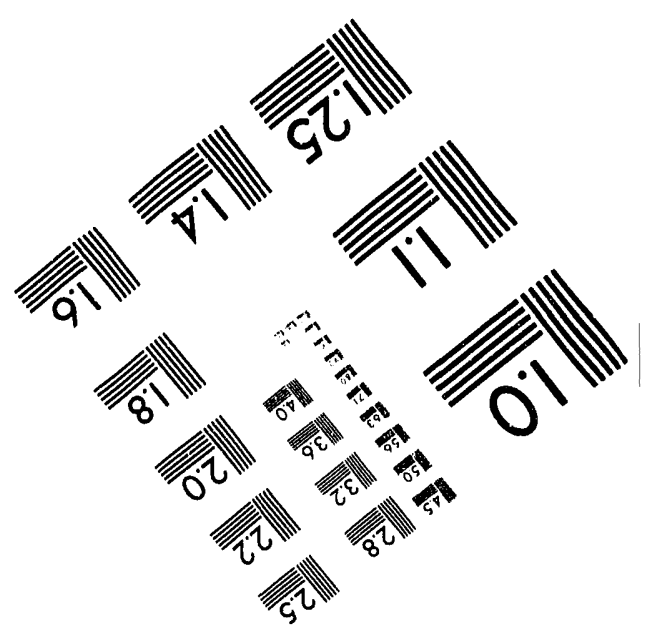



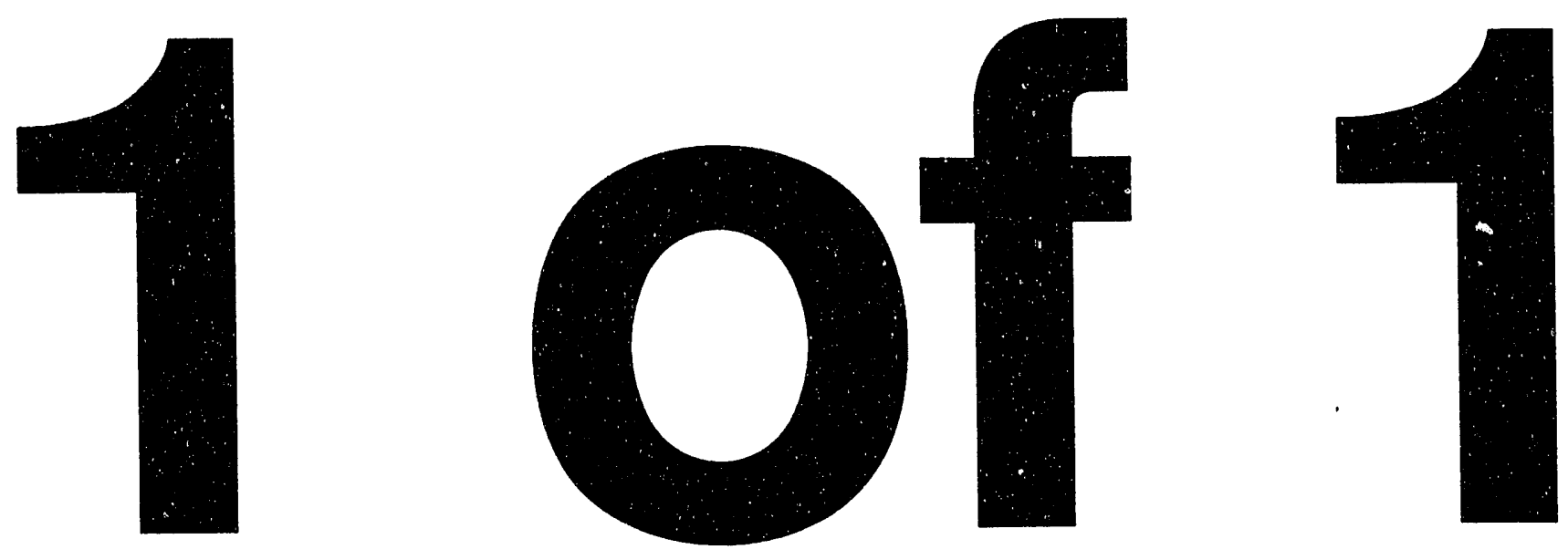


\section{BEAM ENERGY MEASUREMENT USING THE ARC BEAM LINE AS A SPECTROMETER}

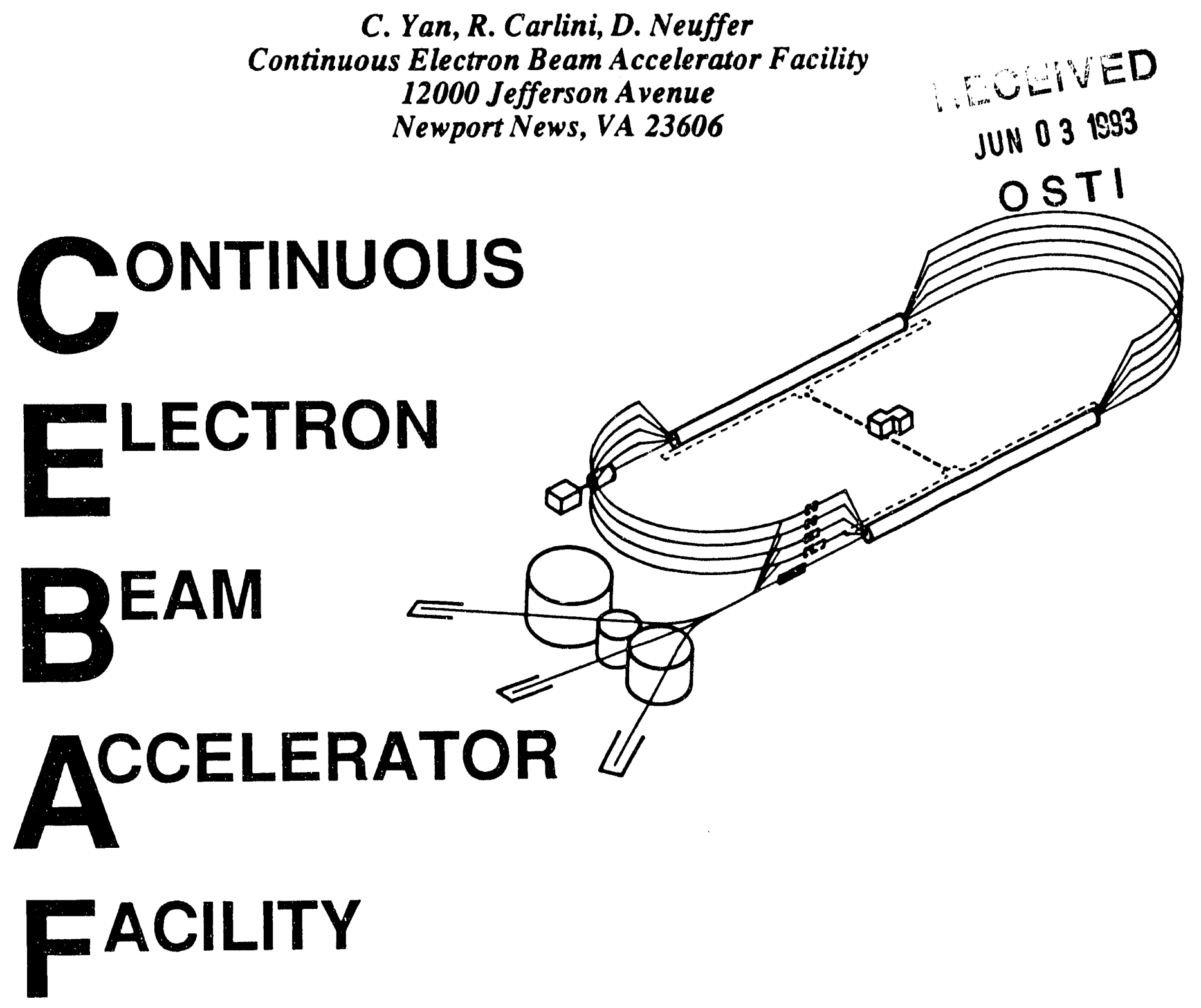

SURA southeastern Universities Research Association Gesifs 
Copies available from:

\author{
Library \\ CEBAF \\ 12000 Jefferson Avenue \\ Newport News \\ Virginia 23606
}

The Southeastern Universities Research Association (SURA) operates the Continuous Electron Beam Accelerator Facility for the United States Department of Energy under contract DE-AC05-84ER40150.

\title{
DISCLAIMER
}

This report was prepared as an account of work sponsored by the United States government. Neither the United States nor the United States Department of Energy, nor any of their employees, makes any warranty, express or implied, or assumes any legal liability or responsibility for the accuracy, completeness, or usefulness of any information, apparatus, product, or process disclosed, or represents that its use would not infringe privately owned rights. Reference herein to any specific commercial product, process, or service by trade name, mark, manufacturer, or otherwise, does not necessarily constitute or imply its endorsement, recommendation, or favoring by the United States government or any agency thereof. The views and opinions of authors expressed herein do not necessarily state or reflect those of the United States government or any agency thereof. 


\title{
BEAM ENERGY MEASUREMENT USING THE ARC BEAM LINE AS A SPECTROMETER
}

\author{
C. Yan, R. Carlini, D. Neuffer \\ Continuous Electron Beam Accelerator Facility \\ 12000 Jefferson Ave., Newport News, VA 23606
}

\section{Abstract}

The use of the Hall $\mathrm{C}$ achromatic arc line as an energy analyser is proposed. It has a dispersion of $12 \mathrm{~cm} / \%$ with all the quadrupoles, sextupoles, and beam correctors switched off. The transverse position and the angle of the beam at the entrance of the arc is precisely measured by a pair of wire scanners spaced by $2.5 \mathrm{~m}$, and the transverse position of the outgonig beam is measured by another pair of wire scanners at the exit of the arc. After the absolute beam energy is measured, the arc will be turned into normal achromat mode by energizing all the elements and the beam position probe located at the mid-point of the arc is used to monitor the beam energy in the operational mode. A complete error analysis shows that an absolute beam energy measurement with $10^{-3}$ accuracy can be achieved. Relative energy measurements at the $10^{-4}$ level are also obtainable.

\section{INTRODUCTION}

The Hall $\mathrm{C}$ beam line is sketched in Figure 1. The arc achromat section of $\mathrm{Hall} \mathrm{C}$ beam line consists of 8 dipole, 12 quads, 8 sextupole, and 8 pairs of beam correctors (vertical and horizonial), which transports the beam with second order achromaticity.

The beam energy measurement by the arc spectrometer was proposed and developed by [1], [2], and [3]. The position and the direction of the beam entering the Hall $C$ arc line are determined at the entrance by a pair of high-resolution harps (wire scanners). The position (and direction) of the beam at the exit of the $34.3^{\circ}$ bend ( 41.6 $m$ downstream from the point of tangency) is determined by another pair of calibrated harp(s). For this procedure only the dipoles are energized. During this absolute measurement all other arc magnetic elements such as quads, sextupoles, beam correctors are off. The current in the calibrated (absolutely) bending magnets is varied to set the position to be along the central ray of the magnets in the arc. With this information the beam momentum can be determined. Thus, this method requires accurate position

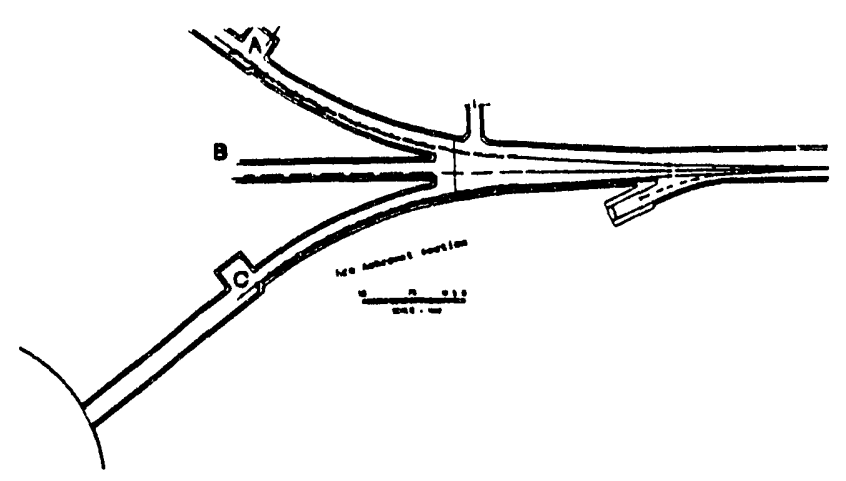

Figure 1: Hall $\mathrm{C}$ beam transport line

(and implicitly direction) measurements at the harps and an accurate determination of the magnetic field integral $\int B d l$ as a function of current $I$ in the arc dipoles.

The quadrupoles and sextupoles are then energized to the values required by the measured energy in the normal achromatic transportation mode. The orbit correctors are activated to center the beam on a beam position measuring device (BPM) situated at the midpoint of the achromat. Under the assumption that the beam momentum is unchanged during this transition period, we can transfer the absolute momentum calibration to the achromatic mode which is very sensitive to relative shifts in the beam momentum. Variations in beam energy can then be measured as variations in beam-position at the midpoint. This achromatic mode will, also, be capable of obtaining relative energy measurements with substantially greater accuracy than the absolute mode.

\section{Basic Optical Performance of t'He ARC SPECTROMETER}

The beam envelope along the Hall $\mathrm{C}$ beam lines is shown in Figure 1. The initial beam conditions at the point of tangency are: $\Delta x=\Delta y=0.01 \mathrm{~cm}, \Delta x^{\prime}=\Delta y^{\prime}=$ $0.01 \mathrm{mr}$. For $\Delta \mathrm{p} / \mathrm{p}=0$, the solid line describes the beam envelope of the normal achromatic transportation lines, 


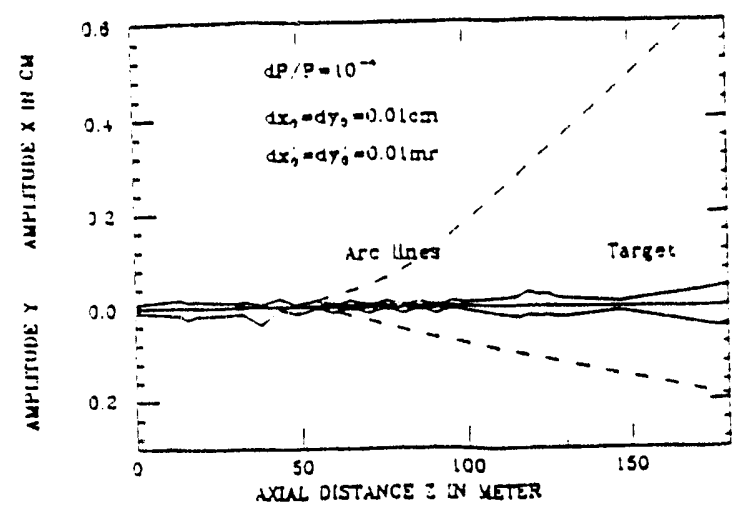

Figure 2: Beam envelope along Hall $\mathrm{C}$ beam lines

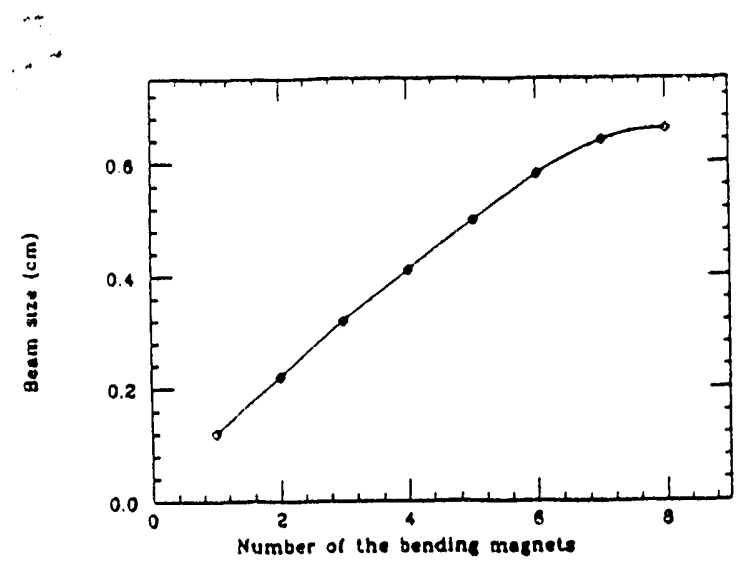

Figure 3: Beam size versus the number of bending magnets

and the dashed line is for the same beam line, but switching off all quadrupoles and sextupoles in the arc section as well is in the final matching section.

Figure 2 and Figure 3 show the beam size and dispersion versus the number of bending magnets in the spectrometer mode. For a $\mathrm{N}$ dipole magriet system the dispersion $\mathrm{D} \sim$ $\mathrm{N}_{\text {dipole }}^{2} \sim \mathrm{L} \phi / 2$, where $\mathrm{L}$ is the total path length of central trajectory, and $\phi$ is the integral deflection angle of the $\mathrm{N}$ dipole magnet system.

The main elements of the first order matrix at the exit of $\mathrm{N}$-dipole systems are listed in Table 1

\section{ERROR ANALYSIS}

The proposed measurement method is planned to obtain absolute energy measurements at the $\delta E / E \approx 10^{-3}$ level. Analyses to support an estimate of errors at this level are required. An initial error analysis was obtained by [3] and the same methods were also used to study variations and changes in the proposed energy measurement configuration. In this section we describe the error analysis methods, including estimates of the expected error sources, and report results of the analyses. The various error sources and their estimated contributions include:

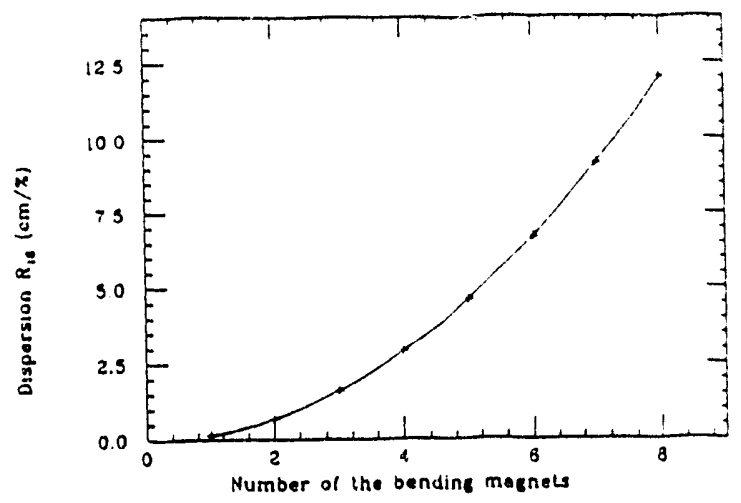

Figure 4: Dispersion versus the number of bending magnets

$\begin{array}{cccccc}\mathrm{N} & \phi\left({ }^{\circ}\right) & \text { Size }(\mathrm{cm}) & R_{11} & R_{12} & D=R_{16}(\mathrm{~m}) \\ & & & & & \\ 1 & \mathbf{4 . 2 8 7 5} & 0.022 & 0.9064 & 2.0234 & -0.1422 \\ 2 & 8.5750 & 0.033 & 1.1015 & 3.0322 & -0.6734 \\ 3 & 12.863 & 0.045 & 1.2965 & 4.0410 & -1.5938 \\ 4 & 17.150 & 0.060 & 1.4916 & 5.0498 & -2.9032 \\ 5 & 21.438 & 0.078 & 1.6866 & 6.0586 & -4.6017 \\ 6 & 25.725 & 0.099 & 1.8817 & 7.0675 & -6.6894 \\ 7 & 30.013 & 0.124 & 2.0767 & 8.0763 & -9.1608 \\ 8 & 34.300 & 0.152 & 2.2718 & 9.0851 & -12.032\end{array}$

Table 1: The major first order matrix elements of $\mathrm{N}$-dipole system 


\section{A. Initial hrrp location and direction}

Surveying errors at each location should be on the order of $100 \mu \mathrm{m}$. However, with an entrance harps separation of $1 \mathrm{~m}$, this implies an initial direction error of $100 \mu \mathrm{rad}$. This $100 \mu \mathrm{rad}$ error translates into a $0.5 \mathrm{~cm}$ position change at the end of the arc, where the dispersion is about $12 \mathrm{~m}$. Thus this error alone would give $\delta \mathrm{p} / \mathrm{p}=0.4 \times 10^{-3} ;$ it is the largest estimated source in [3] analysis.

\section{B. Final harp location}

In the error analysis, it was assumed that a random $20 \mu \mathrm{rad}$ missteering occurs every $10.4 \mathrm{~m}$ (an assumed intermediate monument location), and this accumulates to obtain a dislocation at the end of the $41.6 \mathrm{~m}$ arc. This corresponds to a mislocation of $200 \mu \mathrm{m}$ at every arc cell. It somewhat overshoots the estimate of an rms total error of $200 \mu \mathrm{m}$ displacement at the end of the arc, after smoothing. The total effect on the beam is an rms error of $\delta p / p=0.05 \times 10^{-4}$.

\section{Location, orientation errors, and variations in dipole integrated fields}

Placement errors are assumed to be on the level of $1 \mathrm{~mm}$, they have little effect. A $1 \mathrm{mrad}$ roll error is also included; it changes vertical positions but does not greatly change horizontal (energy measurement plane) locations. A random dipole-to-dipole bend variation of $2.5 \times 10^{-4}$ rms was also assumed. It adds an rms energy error of slightly more than $10^{-3}$.

\section{Quad and steering magnet effect}

In nbsolute energy measurement mode, the quads and steerers are assumed to be off. Remanent fields could add some bending and therefore some error to the energy measurement. In the initial analysis, these are assumed to be negligibly small (contributing errors less than $10^{-4}$ of the dipole bends), and are not explicitly included. In the recent experimental testing, a less than $5 \times 10^{-8}$ remanent field contribution to the $\int B d l$ was found.

\section{E. Beam size effects}

It was assumed that the beam sire at the entrance to the arc was less than $100 \mu \mathrm{m}$ by $10 \mu \mathrm{rad}$. The beam size would then be less than $1.5 \mathrm{~mm}$ at the end of the arc, and would add a width of $10^{-4}$ to the final harp position uncertainty.

\section{F. Field normalization error}

An important error which is not explicitly included in $[3$ ! simulations is the error in mean magnetic field (as a function of current) in the dipoles. This absolute normalization will have to be obtained by a new set of careful absolute measurements on two or a few sample dipoles. Current measurements are absolute at only the 0.01 level. Analysis

$\begin{array}{ccc} & \mathrm{L}=1 \mathrm{~m} & \mathrm{~L}=2.5 \mathrm{~m} \\ \mathrm{~N} & \delta \mathrm{E} / \mathrm{E} & \delta \mathrm{E} / \mathrm{E} \\ & & \\ 1 & 4.0 \times 10^{-3} & 2.3 \times 10^{-3} \\ 2 & 2.1 \times 10^{-3} & 1.0 \times 10^{-3} \\ 4 & 1.14 \times 10^{-3} & 0.44 \times 10^{-3} \\ 8 & 0.50 \times 10^{-3} & 0.23 \times 10^{-3}\end{array}$

Table 3: Error analysis from DIMAD simulation

assumed this absolute calibration could be done to better than the $5 \times 10^{-4}$ level and expected a $2.5 \times 10^{-4}$ error level.

The various error sources were combined with random error generation using transport program DIMAD. DIMAD is an established, "debugged" transport code which is also the basic tool used in the CEBAF transport design. However, it is not optimized for error analysis and it has the disadvantage that every evaluation requires a separate run, and therefore cannot be used to develop largestatistics random variation studies. In [3] analysis, 10 random error seeds were run and obtained error estimates of $3-6 \times 10^{-4}$. Subsequent error analyses obtained the same results shown in Table 2 .

Table 2 indicates that an absolute beam energy measurement at the 1.0 to $1.5 \times 10^{-3}$ level is obtainable.

RAYTRACE program was also used to simulate the error budget. The origin of the output coordinate system was fixed at $z_{D}=0$, where $D$ denotes the final fixed coordinate system in the last element. Different error sources can be simulated in a single run, but no random variation can be used. Results consistent with DIMAD were obtained from RAYTRACE simulation.

\section{Options FOR THE ARC SPECTROMETER}

Following the previous analysis, some variations on the measurement technique were explored. Variation of the placement of the final harp was considered. The $34.3^{\circ}$ arc has 8 dipoles, and the final harp could be located after any one of these. Error analyses for 1, 2, 4, and 8 - dipole configurations were simulated using the same methods and the results are summarized in Table 3

A shorter configuration would permit more accurate alignment. However the dominant error is the initial missteering and the resulting displacement increases linearly with $\mathrm{N}_{D}$, the number of dipoles. The energy-dependent displacement is proportional to the dispersion $D$, which increases as $\mathrm{N}_{D}^{2}$, so the energy error $\delta \mathrm{E} / \mathrm{E}$ decreases as $1 / N_{D}$. Accumulation of random errors also decreases as $1 \checkmark N_{D}$. Thus, the longer arc is favored.

The dominant error is the error in the initial direction, and that error varies inversely as the initial interharp discance Increasing that from the initially proposed value of 


$\begin{array}{lcc}\text { Classified error source } & \text { Figures } & \text { Contribution } \\ \text { Harp fiducialization error } & 50 \mu \mathrm{m} & 3.05 \times 10^{-5} \\ \text { Harp to harp survey error } & 100 \mu \mathrm{m} & 2.29 \times 10^{-5} \\ \text { Survey error between harps } & 200 \mu \mathrm{m} & 2.25 \times 10^{-5} \\ \text { Beam steering accuracy at the entrance } & 100 \mu \mathrm{m} & 3.5 \times 10^{-4} \\ \text { Beam steering accuracy at the exit } & 100 \mu \mathrm{m} & \text { negligible } \\ \Delta \int B d l / \int B d l \text { measurement error } & 2.5 \times 10^{-4} & 2.5 \times 10^{-4} \\ \text { Magnet positioning accuracy } & 1 \mathrm{~mm} & 10^{-4} \\ \text { Magnet orientation accuracy } & 1 \mathrm{mr} & 10^{-4} \\ \text { Magnetic mispowerings (random) } & 10^{-3} & 1.2 \times 10^{-4} \\ \text { Angle kick in the arc } & 0.05 \mathrm{mr} & 1.3 \times 10^{-4} \\ \text { Intermediate monument location accuracy } & 20 \mu \mathrm{rad} & 1.6 \times 10^{-4} \\ \text { Sink of support System } & 2 \mathrm{~mm} \mathrm{in} \mathrm{y} & \text { negligible } \\ \text { Thermal expension of support system } & 2 \mathrm{~mm} \mathrm{in} \mathrm{y} & \text { negligible } \\ \text { Beam initial emittance } & 0.01 \mathrm{~cm}, 0.01 \mathrm{mr} & 3.2 \times 10^{-5} \\ \text { Beam initial energy spread } & 10^{-4} & 10^{-4}\end{array}$

Table 2: Error Sources

$1.0 \mathrm{~m}$ to $2.5 \mathrm{~m}$ (a maximum value with the existing geometry) was considered. The error analyses showed a decrease in the $\mathrm{N}_{D}=8 \delta \mathrm{E} / \mathrm{E}$ error from $0.5 \times 10^{-3}$ to $0.23 \times 10^{-3}$ and a decrease in $\mathrm{N}_{D}=4$ error from $1.14 \times 10^{-3}$ to $0.44 \times 10^{-3}$. The current plan is to increase that interharp distance and obtain the $\sim 2 \times$ reduction in error size.

The error analysis actually uses only three harps. The proposed configuration includes three pairs of harps: pairs at the beginning, center, and end of the arc. The harps at the center provide an energy measurement with transport quads on and the arc tuned to achromatic mode $\left(360^{\circ}\right.$ phase advance), when the dispersion has a $2 \mathrm{~m}$ maximum at the center. This measurement will be calibrated by the proposed absolute energy measurement. The center harps will also provide an additional $\mathrm{N}_{D}=4$ measurement in the absolute energy calibration, which will be an important consistency check. The final harp pair will also provide an independent evaluation of beam direction, and can be used as a consistency check and to reduce steering error (by $\sqrt{ } 2$ ) effects.

The proposed method will be capable of obtaining relative energy measurements with substantially greater accuracy. In that mode the field normalization error is inapplicable and missteering effects are reduced (by the strong focusing and $180^{\circ}$ entrance to arc center phase advance). The dominant error should be harp misalignment and measurement uncertainties. The sum of those errors should be less than $\delta x \sim 0.2 \mathrm{~mm}$. The resulting error in $\delta \mathrm{E} / \mathrm{E}$ (re)ative) will be on the level of $\delta x / D \sim 10^{-4}$.

\section{Field Integral Measurement}

With the increased interharp distance and the above redundant consistency checks, the dominant error souce is expected to be in the absolute calibration of the mean dipole field. A calibration at the $\leq 0.5 \times 10^{-8}$ level is re-

$\begin{array}{lcc}\text { Setting status } & B_{R}(\mathrm{G}) & \int B_{R} d l(\mathrm{G} \mathrm{cm}) \\ \mathrm{B}=800 \mathrm{G}, \mathrm{I}=5 \mathrm{~A} & 2.4 & 43.2 \\ \mathrm{~B}=-800 \mathrm{G}, \mathrm{I}=-5 \mathrm{~A} & -3.99 & 71.82 \\ \begin{array}{l}\text { Swing from } \pm 5 \mathrm{~A} \\ \text { down to 0 }\end{array} & 5.2 & 93.6\end{array}$

Table 4: Remanent field in a steering magnet

quired. Calibration at the $0.25 \times 10^{-3}$ level would permit an absolute energy measurement at the $\delta \mathrm{E} / \mathrm{E} \approx 0.5 \times 10^{-3}$ level.

The $\int B d l$ of the arc bending magnets is measured by a moving wire device with comparison to a reference magnet. The relative accuracy of the moving wire device is about a few $\times 10^{-5}$. In order to obtain precision $\int B d l$ data of the arc magnets, the longitudinal field profile mapping for at least two production BA magnets must be done, and then, from the relative $\int B d l$ data, extract the absolute $\int B d l$ data for each of the arc magnets can be extracted.

In order to know the amplitude of the remanent field of the beam corrector when it is switched off, a CEBAF standard beam corrector (Milhous Control Company : Spool 1 \#45 - coil \#2) was tested by a Kepco four quadrant power supply (BOP 20-20M). Results are displayed in Table 4

The effective length of an arc dipole is $300 \mathrm{~cm}$, and $B=$ $49922 \mathrm{kG}$ at $6 \mathrm{GeV} / \mathrm{c}$. The total $\int B d l$ is about $1.5 \times 10^{B}$ (isuss $\mathrm{cm}$. Therefore, when a beam corrector is turned off. the catio of the remanent $\int B d l$ of the beam corrector to - mun magnet field is about $4.6 \times 10^{-5}$ at $6 \mathrm{GeV} / \mathrm{c}$ and inot is negligible. 
Material of sensor wire Minimum step size of translation Accuracy of absolute calibration Repeativity of mechanical travel Dynamic range of readout Dynamic range of translation Observable vibration magnitude Maximum linear velosity Resolution per revolution Total number of units
$10 \mu$ tungsten wire $0.6 \mu \mathrm{m}$ (variable) better than $10 \mu \mathrm{m}$ better than $10 \mu \mathrm{m}$

$$
1-200 \mu \mathrm{A}
$$$$
7.63 \mathrm{~cm}
$$

less than $3 \mu \mathrm{m}$

$3.81 \mathrm{~mm} / \mathrm{s}$ (variable) 18 Bit 6

Table 5: Specification of Superharp

\section{Vi. Absolute Beam Position Probe - SUPERHARP}

The proposed method requires a beam position measurement with an accuracy of better than $50 \mu \mathrm{m}$. An upgraded CEBAF harp beam profile monitor ("Superharp") is under development. The absolute beam position readout is realized by a multi-turn encoder which backlashlessly connects to the shaft of stepping motor and provide a parallel binary output with 18 bit resolution. For the beam energy measurement, 6 superharps are required. The basic performance of superharp is listed in Table 5

After the absolute beam energy measurement is completed the arc is tuned to the normal achromatic mode. The arc achromat provides a very well defind focus near the mid-point where the magnification $M_{x}$ is unit and the dispersion $D$ is $2.078 \mathrm{~m}$. If a $0.01 \mathrm{~cm}$ beam size is assumed at the point of tangency, the energy resolution at the mid-point of the arc is about $10^{-4}$. For a momentum deviation $\delta=0.05$, the corresponding beam displacement at the mid-point is about $1 \mathrm{~mm}$, and that can be accurately measured by the superharp. Therefore, we propose that at the midpoint of the arc achromat, two superharps are placed at the two sides of the central quadrupole for a consistency check of the absolute measurement and also for an on-line relative beam energy measurement on the level of $\delta p / p \sim 10^{-4}$.

\section{SUMMARY}

The results of the simulations shown above indicate that it is possible to make an absolute beam energy measurement to an accuracy of about $10^{-8}$ with the errors discussed above for the surveying with smoothing and assuming the interharp distance is extended from $1 \mathrm{~m}$ to $2.5 \mathrm{~m}$. There is no need to change the hardware or the optical tuning of the achromat in the original beam line design. As the major precision beam position probes the upgrade CEBAF harp - the "Superharp" is tested and completed, a special alignment technique for the superharps must be carefully considered and implemented. At least two of the production

arc dipole magnets must be mapped to obtain an absolute field integral measurement with an accuracy of $2.5 \times 10^{-4}$

\section{REFERENCES}

[1] C. Yan, R. Carlini, J. Napolitano, D. Neuffer. CEBAFR-92-003, March 25, 1992

[2] D. Neuffer, C. Yan, R. Carlini. CEBAF-TN-92-054, November 24, 1992

[3] R.V. Servranckx. CEBAF-TN-92-044, September 29 , 1992 

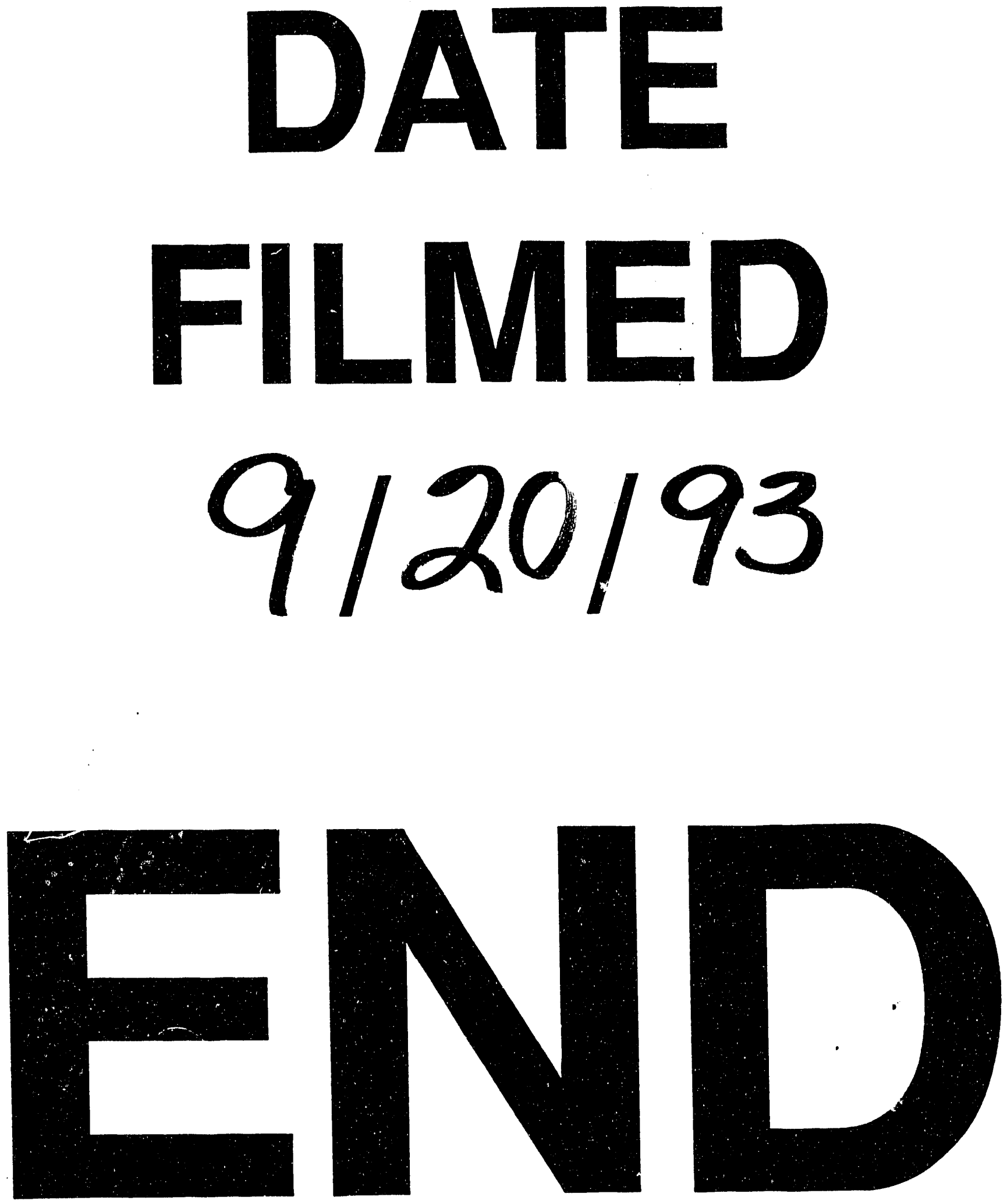
